\title{
Propagation Model for the HVAC Duct as a Communication Channel
}

\author{
Pavel V. Nikitin, Member, IEEE, Daniel D. Stancil, Senior Member, IEEE, Ahmet G. Cepni, Student Member, IEEE, \\ Ozan K. Tonguz, Member, IEEE, Ariton E. Xhafa, Student Member, IEEE, and Dagfin Brodtkorb, Member, IEEE
}

\begin{abstract}
Heating, ventilation, and air conditioning (HVAC) ducts in buildings are typically hollow metal pipes which can be used as waveguides to carry signals and provide the network access to offices. Knowledge of channel properties is crucial to designing such a communication system. This paper presents a propagation model for a straight HVAC duct terminated at both ends. At high frequencies this duct behaves as a multimode waveguide with a transmitting antenna coupling in and a receiving antenna coupling out. We derive a simple analytical expression for the frequency response of this channel using conventional techniques. Experimental data taken on real circular ducts excited by monopole probe antennas confirm theoretical results. This model represents an initial step toward the development of a tool for planning a wireless distribution system using building HVAC ducts.
\end{abstract}

Index Terms-Indoor radio communication, monopole antennas, multimode waveguides, radio propagation, wireless LAN.

\section{INTRODUCTION}

$\mathbf{T}$ HERE are a few different ways to provide network access inside a large office building or a residential house. High-speed connection can be provided by Ethernet line or fiber optics, which may be a costly option. Wireless is an attractive alternative. However, design of a wireless network in large buildings is still a difficult task [1], [2] due to the nature of an indoor propagation [3], [4]. As an alternative, existing infrastructures, such as powerlines [5], [6] or phonelines [7], can be used for data communications. Recently, Stancil and coworkers proposed to use HVAC ducts for signal distribution inside buildings [8], [9]. HVAC ducts present a new communication channel with potentially high data transmission capacity.

Usually, HVAC duct system is a network of interconnected hollow metal pipes of rectangular or circular cross section. An example of a typical duct used in the U.S. is a cylindrical steel pipe of approximately $30 \mathrm{~cm}$ in diameter. This duct behaves as a multimode waveguide when driven at Industrial, Scientific, and Medical (ISM) band frequencies (902-928

Manuscript received November 19, 2001; revised April 3, 2002.

P. V. Nikitin was with the Department of Electrical and Computer Engineering, Carnegie Mellon University, Pittsburgh, PA 15213-3890 USA. He is now with the Department of Electrical Engineering, University of Washington, Seattle, WA 98195-2500 USA (e-mail: nikitin@ee.washington.edu).

D. D. Stancil, A. G. Cepni, O. K. Tonguz, and A. E. Xhafa are with the Department of Electrical and Computer Engineering, Carnegie Mellon University, Pittsburgh, PA 15213-3890 USA (e-mail: stancil@andrew.cmu.edu; acepni@andrew.cmu.edu; tonguz@andrew.cmu.edu; axhafa@andrew.cmu. edu).

D. Brodtkorb is with the Asea Brown Bovari Corporate Research (ABB), Bergerveien 12, N-1735 Billingstad, Norway (e-mail: dagfin. brodtkorb@no.abb.com).

Digital Object Identifier 10.1109/TAP.2003.811491

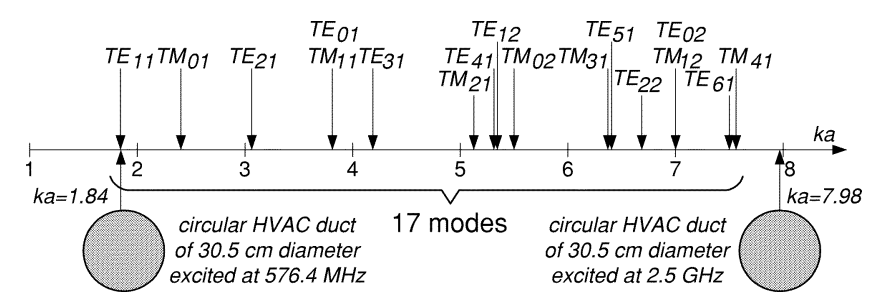

Fig. 1. Modes in a circular HVAC duct.

MHz, 2.4-2.4835 GHz, 5.15-5.35 GHz, 5.725-5.825 GHz). At $2.5 \mathrm{GHz}$, a $30.5-\mathrm{cm}$ diameter circular duct can have 17 propagating modes whose cutoff frequencies are shown in Fig. 1 (the horizontal axis is a unitless parameter $k a$, where the free-space propagation constant $k=2 \pi / \lambda, \lambda$ is the wavelength, and $a$ is the duct radius).

Using multimode waveguides for communications was proposed in the early 1950s [10], [11]. Experimental techniques for characterizing the mode content in multimode waveguides were widely studied in the 1950s and 1960s by Beck [12], Forrer and Tomiyasu [13], Lewis [14], Levinson and Rubinstein [15], and others. One efficient way of coupling in and out of a microwave guide is a monopole probe fed by a coaxial cable. This technique has been mentioned as early as in the late 1940s by Marcuvitz [16], Marchand [17], and Montgomery, Dicke, and Purcell [18]. Most researchers were interested in equivalent impedance or current distribution on such probes. Self-impedance of a coaxially fed probe coupled into a waveguide was first found by Lewin [19] and Collin [20], and then studied extensively by Williamson [21], Rollins [22], Jarem [23], Liang and Zaki [24], and Lee and Yung [25]. The mutual impedance of two probes in an infinite rectangular waveguide was found by Ittipiboon and Shafai [26] using a vector potential. Wang [27], [28] found the mutual impedance of two infinitely thin probes in semi-infinite rectangular and circular waveguides for the multimode case using a general dyadic Green's function. Li and coworkers [29], [30] analyzed the mutual impedance of two probes in an infinite rectangular waveguide with a dielectric discontinuity.

From a communications point of view, we are interested in the frequency response of the channel between two probes coupled into an HVAC duct system rather than in their mutual impedance. It is known that a communication channel can be completely characterized by its frequency response, i.e. transfer function. The latter can in principle be found via mutual impedance. However, mutual impedance formulas derived by the authors mentioned above are very complex even for special cases considered. For the purposes of design of a wireless HVAC distribution system, it is highly desirable 


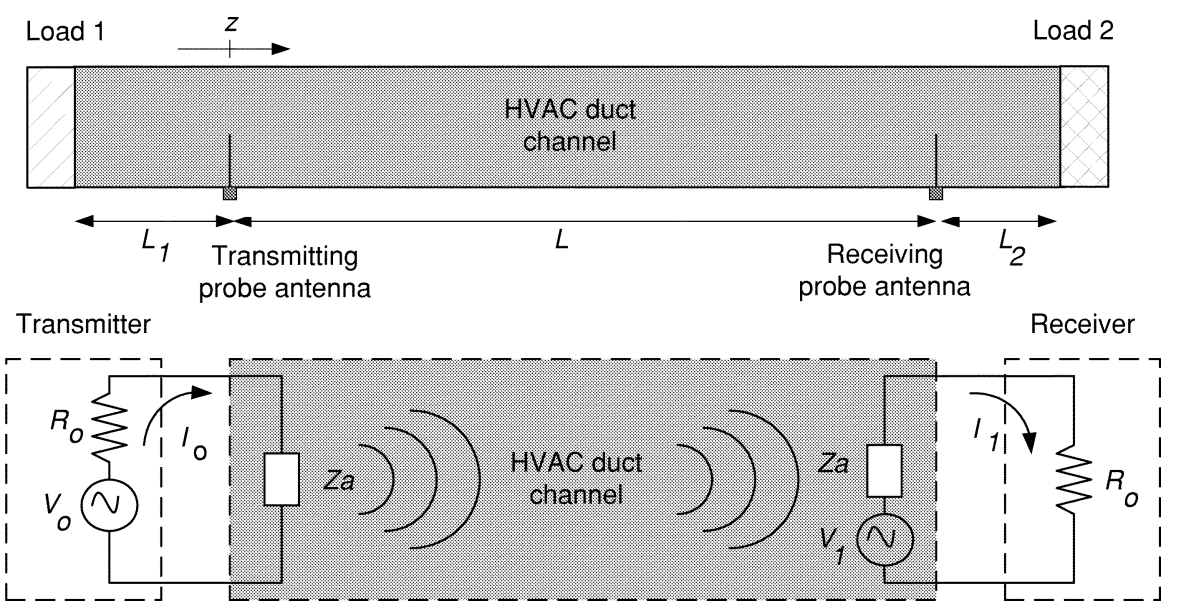

Fig. 2. Transmitting and receiving probe antennas in a straight HVAC duct with different loads on both duct ends (top) and the equivalent circuit model (bottom).

to have a simple analytical model. This model must be valid for ducts of different cross-sections and allow to investigate easily the frequency response dependence on such parameters as antenna geometry, transmitter-receiver separation distance, duct cross-section size, conductivity of the duct material, reflection coefficient of the terminated duct ends, etc. Below we explicitly derive such a model for the HVAC duct channel in the case of a straight multimode duct terminated on both ends, with a transmitting probe antenna coupling in and a receiving probe antenna coupling out.

Section II describes the propagation model. Comparison of theoretical and experimental results is presented in Section III. Section IV contains the discussion of the model and its implications. Conclusions are given in Section V.

\section{PRopagation Model}

Consider a straight HVAC duct of rectangular or circular cross-section, made of metal and terminated at each end as shown in Fig. 2. Two monopole probe antennas provide the coupling. Such a duct is a double-probe waveguide with a number of propagating modes $N$ determined by the operating frequency and the waveguide dimensions. Assume that termination loads 1 and 2 have respective reflection coefficients $\Gamma_{1 n}$ and $\Gamma_{2 n}$ for waveguide mode $n$, which can be frequency-dependent. The distance between the transmitter and the receiver is $L$, and the respective distances to the terminated ends are $L_{1}$ and $L_{2}$.

For a thin monopole probe, a sinusoidal current distribution along the probe length can be assumed [25], [27], [31]

$$
\vec{J}=I_{o} \vec{j}=I_{o} \delta(\zeta) \frac{\sin k(l-\xi)}{\sin k l} \vec{\xi}
$$

where $I_{o}$ is the current amplitude at the antenna terminals, $\vec{j}$ is the normalized current distribution on the antenna, $\zeta$ is the radial coordinate transversal to the probe, $l$ is the probe length, and $\xi$ is the coordinate along the antenna length $(0 \leq \xi \leq l)$.

The current in the transmitting probe radiates in the waveguide and excites electromagnetic (EM) waves travelling for- ward and backward. The electric field $\vec{E}$ of these waves can be expressed in terms of normal waveguide modes as

$$
\vec{E}=\sum_{n=1}^{N} C_{n} \vec{e}_{n} e^{ \pm \gamma_{n} z}
$$

where $\vec{e}_{n}$ is the normalized electric field of the mode $n, C_{n}$ is the mode excitation coefficient, $\gamma_{n}=\alpha_{n}+j \beta_{n}$ is the complex propagation constant, $\alpha_{n}$ is the attenuation constant, and $\beta_{n}$ is the propagation constant. Above, we assumed that the antenna radiates in a waveguide with matched loads and thus neglected the effect of EM fields, reflected from the ends, on the transmitting antenna. We also considered only propagating (nonevanescent) modes.

Fig. 2 also shows the equivalent circuit model, where $Z_{a}$ is the antenna impedance, $V_{o}$ is the voltage of the transmitter source, and both transmitter and receiver are assumed, for simplicity, to have the same internal impedance (resistance) $R_{o}$ and be connected to the probe antennas by matched cables. The current in the transmitting antenna then is

$$
I_{o}=\frac{V_{o}}{R_{o}+Z_{a}}
$$

Using a standard microwave technique for probe-waveguide coupling described, see Collin [20] and Pozar [32], we can calculate mode coefficients to be

$$
C_{n}=-\frac{I_{o}}{4 p_{n}} \int_{o}^{l} \vec{e}_{n} \cdot \vec{j} d \xi
$$

where $p_{n}$ is the normalized power carried by mode $n$ in one direction and the integral is taken over the length of the probe.

The EM field at the receiving antenna can be decomposed into the sum of normal modes. Assume that the mode coefficients at the plane of the receiving probe antenna are $C_{n}^{\prime}$. The field of mode $n$ induces such a current $I_{n}^{\prime}$ in the receiving probe that the tangential electric field due to mode $n$ is zero at the probe surface. If the receiving antenna is identical to the transmitting antenna, then this current can be found from the time reversal and reciprocity principle [18] as

$$
I_{n}^{\prime}=-I_{o} \frac{C_{n}^{\prime}}{C_{n}}
$$




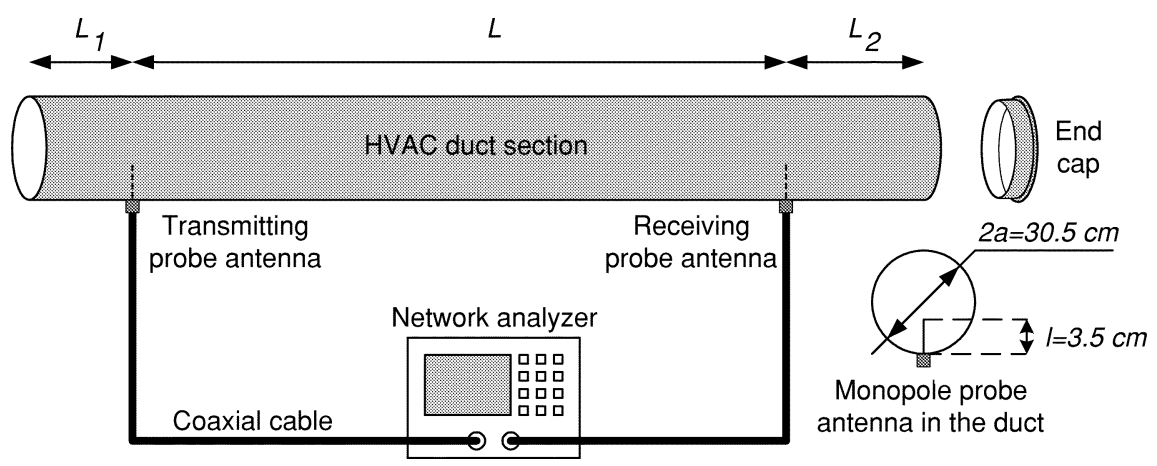

Fig. 3. Experimental setup.

This current causes voltage $V_{n}^{\prime}$ to appear on the receiving antenna. The total voltage on the receiving antenna is

$$
V_{1}=\sum_{n=1}^{N} V_{n}^{\prime}=\sum_{n=1}^{N} I_{n}^{\prime} Z_{n}
$$

where $Z_{n}$ is the antenna impedance due to mode $n$ and the summation is performed over all propagating modes (evanescent modes are neglected since antennas are assumed to be far apart). The total average power dissipated at the receiver load is given by

$$
P_{r}=\left|\frac{V_{1}}{R_{o}+Z_{a}}\right|^{2} \frac{R_{o}}{2} .
$$

Combining (3), (5), (6), and (7) allows one to express $P_{r}$ as

$$
P_{r}=\frac{\left|V_{o} \sum_{n=1}^{N} Z_{n} \frac{C_{n}^{\prime}}{C_{n}}\right|^{2}}{2\left|R_{o}+Z_{a}\right|^{4}} R_{o} .
$$

Now let us find $C_{n}^{\prime}$ in terms of $C_{n}$. Each mode will experience multiple reflections due to reflections from the loads on terminated duct ends. The sum of mode coefficients at the receiving antenna is an infinite geometric series which converges because of the conductor wall loss and the loss at each reflection. Assume that no mode conversion takes place (modes do not mix which means that the summation can be performed separately for each mode). One can easily identify four main terms in a series and their periodical reflections as

$$
\begin{gathered}
C_{n}^{\prime}=C_{n}\left(e^{-\gamma_{n} L}+\Gamma_{1 n} e^{-\gamma_{n}\left(L+2 L_{1}\right)}+\Gamma_{2 n} e^{-\gamma_{n}\left(L+2 L_{2}\right)}\right. \\
\left.+\Gamma_{1 n} \Gamma_{2 n} e^{-\gamma_{n}\left(L+2 L_{1}+2 L_{2}\right)}\right)\left[1+q+q^{2}+q^{3}+\ldots\right]
\end{gathered}
$$

where $q=\Gamma_{1 n} \Gamma_{2 n} e^{-2 \gamma_{n}\left(L+L_{1}+L_{2}\right)}$. After some algebra, we obtain

$$
C_{n}^{\prime}=C_{n} e^{-\gamma_{n} L} \frac{\left(1+\Gamma_{1 n} e^{-2 \gamma_{n} L_{1}}\right)\left(1+\Gamma_{2 n} e^{-2 \gamma_{n} L_{2}}\right)}{1-\Gamma_{1 n} \Gamma_{2 n} e^{-2 \gamma_{n}\left(L+L_{1}+L_{2}\right)}} .
$$

When experimentally characterizing the communication channel response, the network analyzer measurement of the channel loss is typically done with respect to the loss in a reference channel. If transmitter and receiver are connected directly by a lossless matched cable, the reference measured power is

$$
P_{\text {ref }}=\frac{\left|V_{o}\right|^{2}}{8 R_{o}}
$$

The frequency response magnitude can then be determined from

$$
|H(f)|^{2}=\frac{P_{r}}{P_{\text {ref }}} .
$$

Combining (8), (10), and (11) allows one to obtain the final expression for the magnitude of a frequency response

$$
\begin{aligned}
|H(f)| & =\frac{2 R_{o}}{\left|R_{o}+Z_{a}\right|^{2}} \mid \sum_{n=1}^{N} Z_{n} e^{-\gamma_{n} L} \\
& \times \frac{\left(1+\Gamma_{1_{n}} e^{-2 \gamma_{n} L_{1}}\right)\left(1+\Gamma_{2_{n}} e^{-2 \gamma_{n} L_{2}}\right)}{1-\Gamma_{1_{n}} \Gamma_{2_{n}} e^{-2 \gamma_{n}\left(L+L_{1}+L_{2}\right)}} \mid .
\end{aligned}
$$

where impedances $Z_{a}$ and $Z_{n}$ are computed as shown in the Appendix. Expressions for attenuation and propagation constants, needed to compute $\gamma_{n}$, are well known for rectangular and circular waveguides (see, e.g., Collin [20]).

\section{COMPARISON WITH EXPERIMENT}

To validate the model, we performed measurements on a straight section of a circular HVAC duct $30.5 \mathrm{~cm}$ in diameter made of galvanized steel. The experimental setup is shown in Fig. 3. The antennas were thin copper monopole probes $3.5-\mathrm{cm}$ long and $1 \mathrm{~mm}$ in diameter, set on the straight line along the duct length. We used an Agilent E8358A network analyzer with an internal impedance of $50 \mathrm{Ohms}$ to measure the frequency response between the probes in the $2.4-2.5 \mathrm{GHz}$ band.

Fig. 4 shows the experimental frequency response for a circular duct with open ends (approximation of matched loads). The theoretical frequency response was computed for the case of a duct with matched loads on both ends $(\Gamma=0)$. In our model, we used the radiation resistances given by (A.5) and (A.6). The frequency response shape (number of nulls, their depth and position) depends on the excited mode distribution, the distance between the antennas, and the distance to terminations, if any. Recall from Fig. 1 that up to 17 modes can propagate in such a system. The three most significantly excited modes in this geometry are $T E_{61}(R=16.5 \mathrm{Ohm}), T E_{51}(R=8.6 \mathrm{Ohm})$, $T E_{41}(R=3.5 \mathrm{Ohm})$. It is mostly the interference between these three modes that determines the specific locations of peaks and nulls in Fig. 4. Adding more modes increases the accuracy of the theoretical curve. In the presence of only one mode, the frequency response would be very flat.

Fig. 5 shows the experimental frequency response for a circular duct with one end open and the other end covered by a 


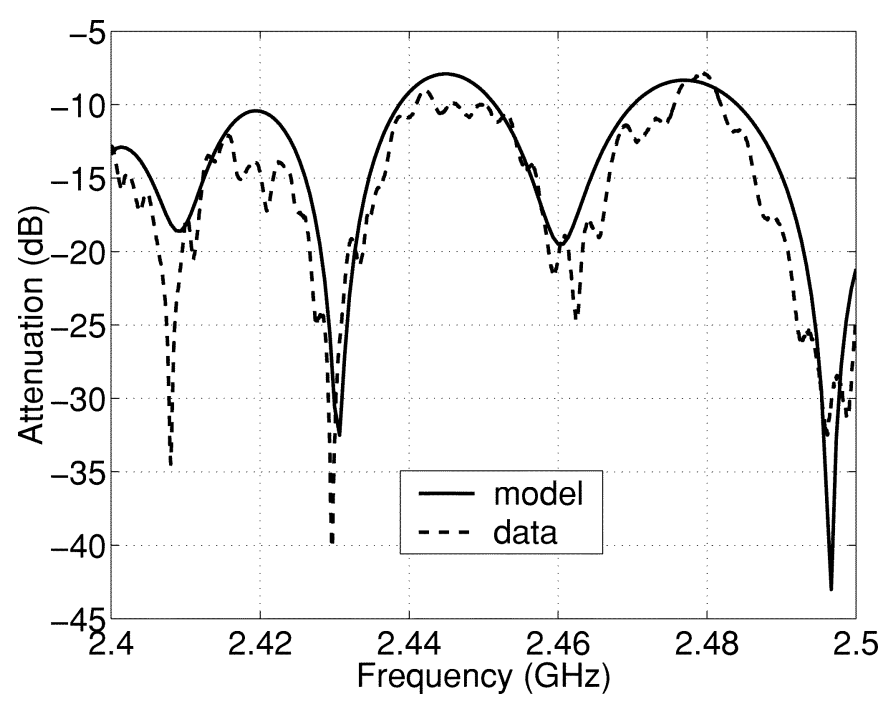

Fig. 4. Theoretical frequency response (solid line) for a circular duct with two matched ends $(\Gamma=0)$ and experimental frequency response (dashed line) for a circular duct with two open ends ( $2 a=30.5 \mathrm{~cm}, L=4.57 \mathrm{~m}, L_{1}=0.25 \mathrm{~m}$, $\left.L_{2}=10.39 \mathrm{~m}, l=3.5 \mathrm{~cm}\right)$.

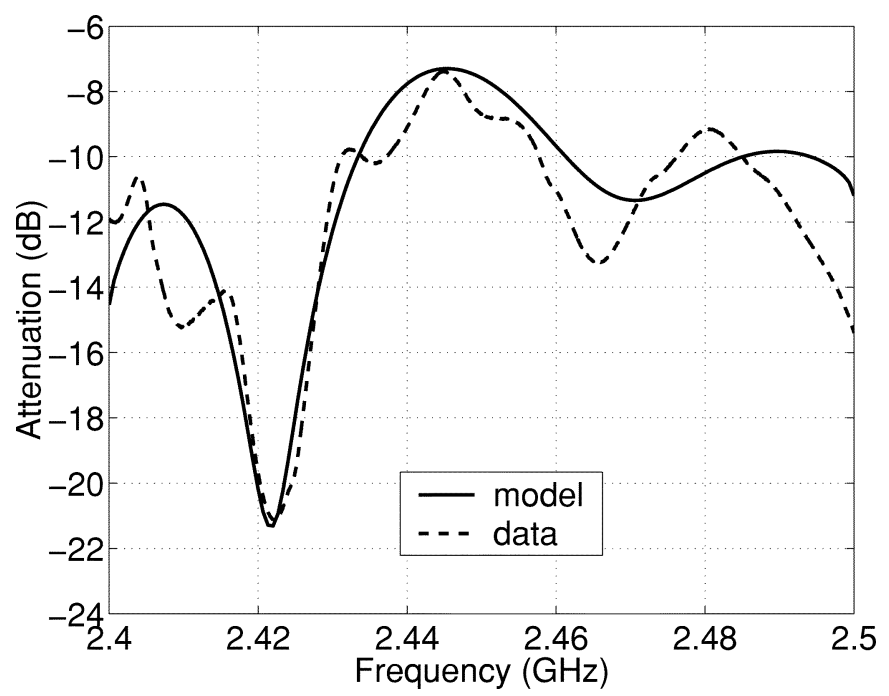

Fig. 5. Theoretical frequency response (solid line) for a circular duct with one matched load $(\Gamma=0)$ and one short-circuit load $(\Gamma=-1)$ and experimental frequency response (dashed line) for a circular duct with one end open and another end covered by a metal cap $\left(2 a=30.5 \mathrm{~cm}, L=2.43 \mathrm{~m}, L_{1}=\right.$ $\left.0.315 \mathrm{~m}, L_{2}=0.315 \mathrm{~m}, l=3.5 \mathrm{~cm}\right)$

metal cap. The theoretical frequency response was computed for the case of a duct with matched load on one end $(\Gamma=0)$ and a short-circuit load on the other end $(\Gamma=-1)$. Again, the specific locations of nulls and peaks can be reproduced by considering only three most excited modes and their reflections from the short-circuited end.

It can be seen that the experimental and theoretical curves are in good agreement. The theoretical curve reproduces all major maxima and minima observed in the experiment. Small scale variations observed on the experimental curve are due partially to mode reflections from open ends and partially to surface and shape imperfections of the circular HVAC duct used for measurements.

\section{DISCUSSION}

Equation (13) specifies the magnitude of a frequency response of the HVAC duct channel. It is presented in a form that is valid for any antenna in a duct of an arbitrary cross-section. The dependence on antenna parameters is contained in the impedances $Z_{n}$ of the waveguide modes. For thin probe antennas these parameters are probe antenna length and its relative orientation. Radiation resistances of probes in rectangular or circular waveguides can be derived analytically (see Appendix). For more complicated antenna types (e.g. post-like structure) radiation resistances have to be computed using other methods, such as a wire-mesh model. Dependence on the waveguide cross-section shape and size (which define the eigenfunctions) is contained both in the impedances $Z_{n}$ and the complex propagation constants $\gamma_{n}$. Dependence on the waveguide wall conductivity is contained only in the complex propagation constants $\gamma_{n}$.

To analyze the propagation characteristics of the HVAC duct system constructed from ducts of a certain type and for specific types of antennas, $Z_{n}$ and $\gamma_{n}$ have to be computed or measured only one time. Once they are determined, the dependence of the frequency response on separation distance $L$, distance to terminations $L_{1}$ and $L_{2}$, reflection coefficients $\Gamma_{1_{n}}$ and $\Gamma_{2_{n}}$ of terminations, and impedance of the transmitter and receiver $R_{o}$ can easily be explored. This capability is especially useful for optimal system design purposes which may require multiple computations of the transfer function. We analyzed identical transmitting and receiving monopole probe antennas but the resulting formula can be modified to account for antennas of different geometry.

A promising use of the HVAC communication channel is providing high-speed network access to the offices in a large building. The HVAC channel is practical and economically viable since it uses an already existing infrastructure. The capacity of this channel depends on the coherence bandwidth. For the frequency response shown in Fig. 4, the coherence bandwidth (50\% signal correlation) is $11 \mathrm{MHz}$. The coherence bandwidth generally reduces with an increase in transmitter-receiver distance due to interference between multiple propagating modes and their reflections in the duct system. The optimal transmission scheme under these circumstances is multicarrier modulation. The maximum possible data rate depends on specific coding, modulation, and equalization schemes and can potentially far exceed the maximum achievable data rate over phonelines and powerlines [9], [33], while providing efficient RF distribution for wireless LANs.

In derivation of the model, several approximations were made which allowed us to obtain a closed-form expression. The rigorous treatment of this problem would involve finding a waveguide Green's function and matching the boundary conditions on the probes and on the duct ends. Real HVAC ducts are not manufactured and joined together with the same degree of precision as microwave guides, so some degree of parameter uncertainty will always be present. However, we feel that the agreement between the model and experimental results presented here is sufficiently good to use the model for estimating the quality of the straight duct communication 
channel. To further improve the agreement between curves presented in Figs. 4 and 5, exact mode reflection coefficients for an open-ended circular waveguide need to be computed, but this task is beyond the scope of the present paper.

\section{CONCLUSION}

HVAC ducts are an attractive medium to use for high-speed communications inside buildings. At high frequencies ducts behave as multimode waveguides which can carry EM waves. To design a wireless HVAC distribution system, a knowledge about the channel is needed. It is highly desirable to have a simple analytical model for the channel. In this paper, we presented an approximate, closed-form, propagation model for the straight HVAC duct channel in the form of a multimode waveguide excited by probe antennas.

We performed experimental measurements to validate our model and found that they confirmed our theoretical results. While in this paper we considered an HVAC channel in the form of a straight duct, the real HVAC duct system is much more complicated, as it may contain tapers, bends, T-junctions, etc. Efficient modeling of RF propagation in such a system is a challenging task. Our model should be perceived as a first step toward predicting the radio coverage in ducts when designing an HVAC wireless distribution system.

\section{APPENDIX}

The antenna impedance $Z_{a}$ and impedance $Z_{n}$ due to mode $n$, which are used in (13), include both radiation resistance and reactance

$$
\begin{aligned}
& Z_{a}=\sum_{n=1}^{N} Z_{n}=R_{a}+j X_{a} \\
& Z_{n}=R_{n}+j X_{n} .
\end{aligned}
$$

The radiation resistance $R_{a}$ is resistance that would consume the same amount of power that is radiated by the antenna into the duct in all modes, if inserted in place of the antenna. Because normal modes in a waveguide are mutually orthogonal, the radiation resistance $R_{a}$ is a linear sum of radiation resistances of all propagating modes

$$
R_{a}=\sum_{n=1}^{N} R_{n}
$$

The resistance $R_{n}$ due to mode $n$ is defined similarly and determines how well an antenna couples into a specific mode. It can be found from

$$
\frac{I_{o}^{2} R_{n}}{2}=2\left|C_{n}\right|^{2} p_{n}
$$

For an antenna in a waveguide with matched loads, the radiation resistance is determined by waveguide eigenfunctions and antenna geometry. We calculated the radiation resistance due to
$T E_{\mathrm{nm}}$ and $T M_{\mathrm{nm}}$ modes for a thin monopole probe in a circular multimode waveguide with matched loads to be

$$
\begin{aligned}
R_{T E_{\mathrm{nm}}}= & \eta \cdot \frac{k}{\beta_{T E_{\mathrm{nm}}}} \\
& \cdot \frac{\left[\int_{1-\frac{l}{a}}^{1} J_{n}\left(p_{\mathrm{nm}}^{\prime}\right) n \xi^{-1} \sin \left[k a\left\{\frac{l}{a}-1+\xi\right\}\right] d \xi\right]^{2}}{\pi \sin ^{2} k l J_{n}^{2}\left(p_{\mathrm{nm}}^{\prime}\right)\left(p_{\mathrm{nm}}^{\prime 2}-n^{2}\right)}, \\
R_{T M_{\mathrm{nm}}}= & \eta \cdot \frac{\beta_{T M_{\mathrm{nm}}}}{k} \\
& \frac{\left[\int_{1-\frac{l}{a}}^{1} J_{n}^{\prime}\left(p_{n m} \xi\right) \sin \left[k a\left\{\frac{l}{a}-1+\xi\right\}\right] d \xi\right]^{2}}{\pi \sin ^{2} k l J_{n}^{\prime 2}\left(p_{\mathrm{nm}}\right)}
\end{aligned}
$$

where $\eta$ is the free space impedance, $\beta_{T E_{\mathrm{nm}}}$ and $\beta_{T M_{\mathrm{nm}}}$ are mode propagation constants in the waveguide, $J_{n}(x)$ is the Bessel function of the first kind of order $n, J_{n}^{\prime}(x)$ is its derivative, $p_{\mathrm{nm}}^{\prime}$ is the $m$ th zero of $J_{n}^{\prime}(x)$, and $p_{\mathrm{nm}}$ is the $m$ th zero of $J_{n}(x)$. The above expressions contain definite integrals which are unitless and can easily be computed numerically for any given combination of parameters. Equation (A.5) reduces to the one derived by Lee [25] for the $T E_{11}$ mode case.

Antenna reactance $X_{a}$ is also found as a linear sum

$$
X_{a}=\sum_{n=1}^{N} X_{n}
$$

where reactance $X_{n}$ due to mode $n$ can be found via the Hilbert transform if the resistance $R_{n}$ is known

$$
X_{n}(\omega)=\frac{1}{\pi} \int_{-\infty}^{+\infty} \frac{R_{n}(y)}{\omega-y} d y
$$

We computed the reactance via the Hilbert transform using a series expansion for the band-limited case as explained in detail in [34].

\section{ACKNOWLEDGMENT}

The authors are grateful to the editor and to the two anonymous reviewers whose comments helped to significantly improve this manuscript. P. Nikitin would also like to thank his Ph.D. dissertation committee members Prof. J. F. Hoburg, Prof. T. E. Schlesinger, and Professor R. Nicolaides for their valuable and helpful feedback, suggestions, and discussions.

\section{REFERENCES}

[1] D. F. Bantz and F. J. Bauchot, "Wireless LAN design alternatives," IEEE Network, vol. 8, pp. 43-53, Mar.-Apr. 1994.

[2] A. Hills, "Large-scale wireless LAN design," IEEE Commun. Mag., vol. 39, pp. 98-107, Nov. 2001.

[3] T. S. Rappaport, "Indoor radio communications for factories of the future," IEEE Commun. Mag., vol. 27, pp. 15-24, May 1989.

[4] H. Hashemi, "The indoor radio propagation channel," Proc. IEEE, vol. 81, pp. 943-968, July 1993.

[5] W. Gibbs, "The network in every room," Sci. Amer, pp. 38-43, Feb. 2002.

[6] D. Clark, "Powerline communications: finally ready for prime time?," IEEE Internet Comput., vol. 2, pp. 10-11, Jan.-Feb. 1998. 
[7] E. H. Frank and J. Holloway, "Connecting the home with a phone line network chip set," IEEE Micro, vol. 20, no. 2, pp. 27-37, Mar-Apr. 2000.

[8] D. D. Stancil and C. P. Diehl, "Wireless signal distribution in a building HVAC system ," U.S. Patent 5994 984, Nov. 30, 1999.

[9] D. D. Stancil, O. K. Tonguz, A. Xhafa, A. Cepni, P. Nikitin, and D. Brodtkorb, "High-speed internet access via HVAC ducts: a new approach," in Proc. IEEE Global Telecommunications Conf. (GLOBECOM'01), vol. 6, San Antonio, TX, Nov. 2001, pp. 3604-3607.

[10] S. E. Miller and A. C. Beck, "Low-loss waveguide transmission," in Proc. Institute Radio Engineers (IRE), vol. 41, 1953, pp. 348-358.

[11] S. E. Miller, "Waveguide as a communication medium," Bell Syst. Tech. $J .$, vol. 33, no. 6, pp. 1209-1265, 1954

[12] A. C. Beck, "Measurement techniques for multimode waveguides," IRE Trans. Microwave Theory Tech., pp. 35-42, 1955.

[13] M. P. Forrer and K. Tomiyasu, "Determination of higher order propagating modes in waveguide systems," J. Appl. Phys., vol. 29, pp. 1040-1045, July 1958.

[14] D. J. Lewis, "Mode couplers and multimode measurement techniques," IRE Trans. Microwave Theory Tech., vol. MTT-7, pp. 110-116, Jan. 1959.

[15] D. S. Levinson and I. Rubinstein, "A technique for measuring individual modes propagating in overmoded waveguide," IRE Trans. Microwave Theory Tech., vol. MTT-14, no. 7, pp. 310-322, July 1966.

[16] N. Marcuvitz, Waveguide Handbook. New York: McGraw-Hill, 1948, vol. 10, MIT Rad. Lab., ch. 5.

[17] N. Marchand, Ultrahigh Frequency Transmission and Radiation. New York: Wiley, 1947, ch. 7, pp. 254-257.

[18] C. G. Montgomery, R. H. Dicke, and E. Parcell, Principles of Microwave Circuits. New York: McGraw-Hill, 1948, vol. 8, MIT Rad. Lab., pp. 343-349.

[19] L. Lewin, "A contribution to the theory of probes in waveguide," Proc. Inst. Elect. Eng., vol. 105, pp. 109-116, Oct. 1958.

[20] R. E. Collin, Field Theory of Guided Waves. New York: IEEE Press, 1990, pp. 471-483.

[21] A. G. Williamson, "Coaxially fed hollow probe in a rectangular waveguide," Proc. Inst. Elect. Eng., vol. 132, pp. 273-285, Aug. 1985.

[22] J. M. Rollins and J. M. Jarem, "The input impedance of a hollow-probe fed, semi-infinite rectangular waveguide," IEEE Trans. Microwave Theory Tech., vol. 37, pp. 1144-1146, July 1989.

[23] J. M. Jarem, "Analysis of a probe-metallic sleeve feed system for a rectangular waveguide," in Proc. IEEE Antennas and Propagation Soc. Int. Symp., vol. 2, 1989, pp. 942-945.

[24] J. F. Liang and K. A. Zaki, "Coaxial probe modeling in waveguides and cavities," IEEE Trans. Microwave Theory Tech., vol. 40, no. 12, pp. 2172-2180, Dec. 1992

[25] W. S. Lee and E. K. Yung, "The input impedance of a coaxial line fed probe in a cylindrical waveguide," IEEE Trans. Microwave Theory Tech., vol. 42, pp. 1468-1473, Aug. 1994.

[26] A. Ittipiboon and L. Shafai, "Probe mutual impedance in a rectangular waveguide," IEEE Trans. Microwave Theory Tech., pp. 327-335, Apr. 1985.

[27] B. Wang, "Mutual impedance between probes in a waveguide," IEEE Trans. Microwave Theory Tech., vol. 36, pp. 53-60, Jan. 1988.

[28] - "Mutual impedance between probes in a circular waveguide," IEEE Trans. Microwave Theory Tech., vol. 37, pp. 1006-1012, June 1989.

[29] L. W. Li, P. S. Kooi, M. S. Leong, and T. S. Yeo, "Full-wave analysis of antenna radiation in a rectangular waveguide with discontinuity. II. probe mutual impedance," in Proc. IEEE Conf. Communication Systems, vol. 2, 1994, pp. 464-468.

[30] - "Mutual impedance of two probes located in two different waveguide regions separated by a homogeneous dielectric slab: fullwave analysis," IEE Trans. Microwaves, Antennas and Propagat., vol. 144, no. 5, pp. 329-336, Oct. 1997.

[31] Y. Huang, "Current distribution along a probe in waveguide," in Proc. IEEE Antennas and Propagation Soc. Int. Symp., vol. 1, 1990, pp. 513-516.

[32] D. M. Pozar, Microwave Engineering. New York: Wiley, 1998, ch. 4.

[33] A. E. Xhafa, O. K. Tonguz, A. G. Cepni, D. D. Stancil, P. V. Nikitin, and D. Brodtkorb, "Theoretical estimates of HVAC duct capacity for high-speed internet access," in Proc. IEEE Int. Conf. Communications (ICC'02), vol. 2, New York, May 2002, pp. 936-939.

[34] A. E. Gera, "Linking resistance and reactance via Hilbert transforms," in Proc. IEEE 17th Conf. Electrical and Electronics Engineers in Israel, Ramat-Gan, Israel, May 1991, pp. 141-144.

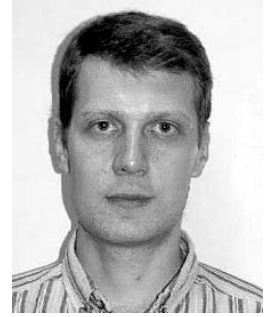

Pavel V. Nikitin (S'98-M'02) was born in Ashkhabad, U.S.S.R., in March 1974. He received the B.S. and M.S. degrees in electrical engineering from Utah State University, Logan, in 1994 and 1998, respectively, the B.S. degree in physics from Novosibirsk State University, Novosibirsk, Russia, in 1995, and the Ph.D. degree in electrical and computer engineering, Carnegie Mellon University (CMU), Pittsburgh, PA, in 2002.

Previously, he was a Research Assistant in the Antenna and Radio Communication Group, Center for Wireless and Broadband Networking, CMU. Currently, he is a Research Associate at the Department of Electrical Engineering at the University of Washington, Seattle. His research interests include applied electromagnetics, wireless communications, and computer-aided design of integrated circuits and systems.

Dr. Nikitin is a member of the Honor Society of Phi Kappa Phi. In 2000, he was a recipient of the ECE Teaching Assistant of the Year Award from CMU.

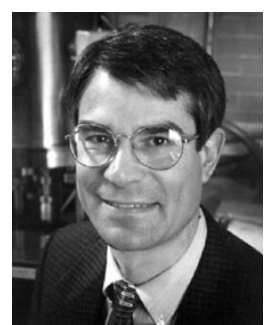

Daniel D. Stancil (S'75-M'81-SM'91) received the B.S. degree in electrical engineering from Tennessee Technological University, Cookeville, in 1976, and the S.M., E.E., and Ph.D. degrees from the Massachusetts Institute of Technology, Cambridge, in 1978, 1979 , and 1981, respectively.

From 1981 to 1986, he was an Assistant Professor of Electrical and Computer Engineering at North Carolina State University, Raleigh. In 1986, he became an Associate Professor at Carnegie Mellon University (CMU), Pittsburgh, PA, where he is currently a Professor in the Department of Electrical and Computer Engineering, leads the Antenna and Radio Communication Group, and heads the newly established Center for Wireless and Broadband Networking. In 1996 he cofounded the Applied Electro-optics Corporation, Pittsburgh, PA

Dr. Stancil received a Sigma Xi Research Award from North Carolina State University in 1985, and was a leader in the development of the CMU Electrical and Computer Engineering Department's Virtual Laboratory which was a finalist for a 1996 Smithsonian Computerworld Award. In 1998, he coreceived a Science Award for Excellence from the Pittsburgh Carnegie Science Center, an IR 100 Award, and a Photonics Circle of Excellence Award for the development and commercialization of electro-optics technology. He is a Past-President of the IEEE Magnetics Society.

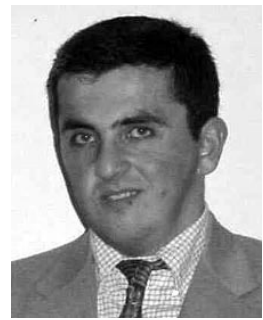

Ahmet G. Cepni (S'01) received the B.S. degree in electrical and electronics engineering and in physics, both from Bogazici University, Istanbul, Turkey, in 2000 and the M.S. degree in electrical and computer engineering from Carnegie Mellon University (CMU), Pittsburgh, PA, in 2002, where he is currently working toward the Ph.D. degree.

Since January 2001, he has been a Research Assistant at the Antenna and Radio Communications Research Group at CMU. He has been working on indoor wireless communications via HVAC duct project. His research interests are intelligent radio architectures, $\mathrm{RF} /$ microwave systems, and propagation models. 


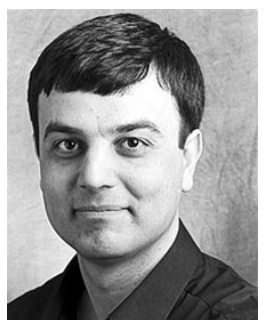

Ozan K. Tonguz (S'86-M'90) was born in Cyprus, in May 1960. He received the B.Sc. degree from the University of Essex, Essex, U.K., in 1980, and the M.Sc. and Ph.D. degrees from Rutgers University, New Brunswick, NJ, in 1986 and 1990, respectively, all in electrical engineering.

From 1988 to 1990, he was with Bell Communications Research (Bellcore), doing research in optical networks and communication systems. In 1990, he joined the State University of New York at Buffalo as an Assistant Professor, where he was granted early tenure and promoted to Associate Professor in 1995, and to Full Professor in 1998. Since 2000, he has been at Carnegie Mellon University (CMU), Pittsburgh, PA, where he is currently a tenured Full Professor in the Department of Electrical and Computer Engineering and a Codirector (Thrust Leader) of the Center for Wireless and Broadband Networking Research. His industrial experience includes periods with Bell Communications Research, CTI Incorporated, Harris RF Communications, Aria Wireless Systems, Clearwire Technologies, Nokia Networks, and Asea Brown Bovari (ABB). He currently serves as a Consultant for several companies, law firms, and government agencies in the U.S and Europe in the broad area of telecommunications and networking. His current research interests are in optical networks, wireless networks and communication systems, high-speed networking, and satellite communications. Author or coauthor of more than 120 technical papers in IEEE journals and conference proceedings, and a book chapter in The Encyclopedia of Electrical and Electronics Engineers (New York: Wiley, 1999), his contributions in optical networks and wireless networks are internationally acclaimed. He has published in the areas of optical networks, wireless communications and networks, and high-speed networking.

Dr. Tonguz currently serves or has served as an Associate Editor for the IEEE TRANSACTIONS ON COMMUNICATIONS, IEEE COMMUNICATIONS MAgAZINE, and IEEE/OSA Journal of LightwaVe TeCHNOLOGY. He was a Guest Editor of the special issue of the IEEE/OSA JOURNAL OF LIGHTWAVE TECHNOLOGY and IEEE JOURNAL ON SELECTED AREAS IN COMMUNICATIONS on Multiwavelength Optical Networks and Technology, published in 1996. In addition, he has served on the Technical Program Committees of several IEEE conferences and symposia in the area of wireless communications and optical networks.

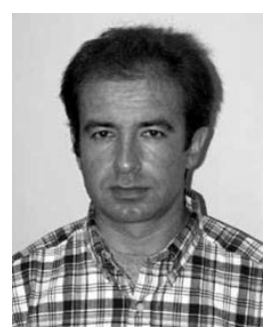

Ariton E. Xhafa (S'98) received the B.S. degree in electrical and electronics engineering and physics from Eastern Mediterranean University, North Cyprus, Turkey, in 1997 and the M.S. degree in electrical engineering from the State University of New York at Buffalo, in 1999. He is currently working toward the Ph.D. degree in the Electrical and Computer Engineering Department at Carnegie Mellon University (CMU), Pittsburgh, PA.

From 2000 until 2001, he was a Visiting Researcher at CMU, doing research on design and performance analysis of communications systems. Since 2002, he has been a Research Assistant at the Telecommunications Research Group at CMU. His current research interests include design and performance analysis of wireless networks, scheduling, resource allocations and queuing theory, and handover management and quality of service in communications networks.

Mr. Xhafa is a Student Member of the IEEE Communications Society, IEEE Vehicular Technology Society, and IEEE Computer Society.

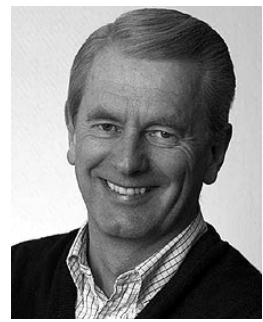

Dagfin Brodtkorb (M'70) was born in Norway, in July 1947. He received the M.S. and the Ph.D. degrees in electrical engineering, both from the Norwegian Institute of Technology, Trondheim, in 1970 and 1976 , respectively.

From 1971 to 1980, he was a Research Engineer and Project Manager with the Norwegian Defense Research Establishment, Kjeller, Norway, where he worked on radar and microwave systems. From 1977 to 1978 , he was a Postdoctoral Researcher at the Massachusetts Institute of Technology, Lincoln Laboratory, Lexington, MA, where he worked on surface acoustic wave (SAW) technology. From 1980 to 1984, he was with A/S Informasjonskontroll, Asker, Norway, as a Manager of the Remote Sensing Group. From 1984 to 1995, he was Managing Director of MIROS A/S, Asker, Norway. Since 1995, he has been with Asea Brown Bovari Corporate Research (ABB), Billingstad, Norway, first as a Senior Research Manager, then as a Technology Program Manager, and as a Department Manager. He is currently a Vice President (Research Director) of the ABB Corporate Research Center in Norway. He is the author of a few technical papers. 